\title{
Galerkin Methods for Second Kind Integral Equations With Singularities
}

\author{
By Ivan G. Graham
}

\begin{abstract}
This paper discusses the numerical solution of Fredholm integral equations of the second kind which have weakly singular kernels and inhomogeneous terms. Global convergence estimates are derived for the Galerkin and iterated Galerkin methods using splines on arbitrary quasiuniform meshes as approximating subspaces. It is observed that, due to the singularities present in the solution being approximated, the resulting convergence may be slow. It is then shown that convergence will be improved greatly by the use of splines based on a mesh which has been suitably graded to accommodate these singularities. In fact, it is shown that, under suitable conditions, the Galerkin method converges optimally and the iterated Galerkin method is superconvergent. Numerical llustrations are given.
\end{abstract}

1. Introduction. In this paper we shall discuss the numerical solution of equations of the form

$$
y(t)=f(t)+\int_{0}^{1} k(t, s) y(s) d s, \quad t \in[0,1],
$$

where $k$ and $f$ are given functions on $[0,1] \times[0,1]$ and $[0,1]$, respectively, and $y$ is the solution to be determined. We abbreviate (1.1) by

$$
y=f+K y,
$$

where $K$ is the integral operator given by

$$
K y(t)=\int_{0}^{1} k(t, s) y(s) d s .
$$

The Galerkin and iterated Galerkin methods are well-established numerical algorithms for the approximate solution of (1.1). It has been shown by Sloan et al., [28], [26], [27], that the iterated Galerkin method provides, in general, a more accurate approximation to $y$ than does the Galerkin method. Accurate quantitative estimates for this improvement in order (or "superconvergence") have been obtained by Chandler [7], [8], [9], for the case when the underlying approximating subspace is a space of splines, and when the kernel $k$, and the inhomogeneous term $f$, are suitably smooth. The aim of this paper will be to obtain quantitative estimates for Galerkin and iterated Galerkin methods, again when splines are used as approximating functions, in the case when $k$ and $f$ may contain certain weak singularities. Such weakly singular equations arise often in practical situations [17], [23], [25].

Received June 23, 1981.

1980 Mathematics Subject Classification. Primary 65R20, 45B05, 45L05.

Key words and phrases. Second kind Fredholm integral equation, weak singularities, Galerkin method, iterated Galerkin method, spline approximation, graded mesh. 
Our first main order of convergence result in Theorem 8 of Section 4. To illustrate the results of this rather general theorem, we use as an example the particular equation

$$
y(t)=t^{\beta}+\int_{0}^{1} m(t, s)|t-s|^{\alpha-1} y(s) d s, \quad t \in[0,1],
$$

where $1>\alpha>0,1>\beta>0$, and $m$ is smooth. Suppose our approximating subspace is a space of continuous splines of order $r$ (i.e., of degree $r-1$ ) defined on a uniform mesh over $[0,1]$, and let $y_{n}^{\mathrm{I}}$ and $y_{n}^{\mathrm{II}}$ denote, respectively, the Galerkin and iterated Galerkin approximants to $y$. Then Theorem 8 predicts that

$$
\left\|y-y^{\mathrm{I}}\right\|_{\infty}=O\left(1 / n^{\gamma}\right)
$$

and

$$
\left\|y-y_{n}^{\mathrm{II}}\right\|_{\infty}=O\left(1 / n^{\gamma+\alpha}\right)
$$

where $\gamma=\min \{\alpha, \beta\}$, and $n+1$ is the number of points in the mesh.

More general error estimates are given in Theorem 8 . However, the illustration given here highlights two important points which are also true in the general case.

(i) The improvement obtained by using $y_{n}^{\mathrm{II}}$ instead of $y_{n}^{\mathrm{I}}$ is $O\left(1 / n^{\alpha}\right)$.

(ii) If either $\alpha$ or $\beta$ is small, then both $y_{n}^{\mathrm{I}}$ and $y_{n}^{\mathrm{II}}$ may converge rather slowly to $y$, regardless of how large $r$ is.

The reason for the phenomenon (ii) is the well-known fact [5], [9], [16], [22], [24], [31], that any weakly singular integral equation, such as (1.3), will, in general, have a nonsmooth solution, and the order of approximation of such a solution using splines on a uniform (or indeed arbitrary) mesh will, in general, be rather low.

This order may be improved, however, if we use a mesh which takes account of the singularities in the solution. In Section 5, we consider a class of equations of which (1.3) is an example and demonstrate how to improve convergence by using an appropriate graded mesh. Our second main order of convergence result is Theorem 10 of Section 5. In particular, this theorem shows that, with a carefully chosen mesh, (1.4) and (1.5) may be improved to

$$
\left\|y-y_{n}^{\mathrm{I}}\right\|_{\infty}=O\left(1 / n^{r}\right)
$$

and

$$
\left\|y-y_{n}^{\mathrm{II}}\right\|_{\infty}=O\left(1 / n^{r+\alpha}\right)
$$

(i.e., $y_{n}^{\mathrm{I}}$ is optimal and $y_{n}^{\mathrm{II}}$ is superconvergent).

The main convergence theory is contained in Sections 4 and 5. In Section 2 we define the Galerkin and iterated Galerkin algorithms and give a résumé of existing convergence results. In Section 3 we present some theoretical tools which are used in Sections 4 and 5. The theory is illustrated in Section 6 by some numerical calculations.

The numerical solution of weakly singular integral equations has recently been the subject of much research activity. For example, Chandler [9], [10], Schneider [25] and Bechlars [5] have studied product integration, using graded meshes to obtain good convergence rates. Vainikko and Uba [32] have done the same for collocation methods. Spence [29] has used extrapolation methods to improve the rate of 
convergence of product integration methods for weakly singular equations. (Lin Qun and Liu Jiaquan [20] have looked at extrapolation methods for equations with Green's function type kernels.) Anselone and Krabs [3] have used a double approximation scheme based on replacing singular functions by bounded approximations, while Anselone [2] has given a theoretical basis for the popular practical technique [23] of subtracting out the singularity from the solution. Delves, Abd-Elal and Hendry [13] have studied ways of making the Galerkin method for weakly singular equations more economical. We also note the extensive treatment in Baker's book [4, Sections 5.3-5.8], where the performance of most of the standard methods, as applied to the numerical solution of weakly singular equations is discussed. Many numerical examples are given there, and also in the reports of Bechlars [5] and Volk [33].

This present paper is an amplified version of a preliminary study made in [17].

2. Methods and Background. We shall let $\mathbf{N}$ denote the positive integers, and $\mathbf{N}_{0}$ denote the nonnegative integers. Let us assume, for the moment, that $K$ is a compact operator on $L_{2}[0,1]$, that $(I-K)^{-1}$ is well defined on $L_{2}[0,1]$, and that $f \in L_{2}[0,1]$. For each $n \in \mathbf{N}$, let $U_{n}$ denote some finite-dimensional subspace of $L_{2}[0,1]$, let $P_{n}$ denote the orthgonal projection of $L_{2}[0,1]$ onto $U_{n}$, and suppose that $\left\|\phi-P_{n} \phi\right\|_{2} \rightarrow$ 0 , as $n \rightarrow \infty$, for every $\phi \in L_{2}[0,1]$. The Galerkin solution of $(1.1), y_{n}^{\mathrm{I}}$, is then defined by the equation

$$
y_{n}^{\mathrm{I}}=P_{n} f+P_{n} K y_{n}^{\mathrm{I}},
$$

and the iterated Galerkin solution, $y_{n}^{\mathrm{II}}$, is obtained by the natural iteration:

$$
y_{n}^{\mathrm{II}}=f+K y_{n}^{\mathrm{I}} \text {. }
$$

For details of the practical computation of these approximate solutions, see Sloan et al. [28]. Applying the operator $P_{n}$ to each side of (2.2), and comparing with (2.1), it follows that

$$
P_{n} y_{n}^{\mathrm{II}}=y_{n}^{\mathrm{I}},
$$

which on substitution into (2.2) gives

$$
y_{n}^{\mathrm{II}}=f+K P_{n} y_{n}^{\mathrm{II}} \text {. }
$$

A proof of Theorem 1 below can be then found in Sloan [26].

TheOREM 1 (SLOAN). For sufficiently large $n, y_{n}^{\text {II }}$ is well defined, and

$$
\left\|y-y_{n}^{\mathrm{II}}\right\|_{2} \leqslant C\left\|K y-K P_{n} y\right\|_{2} \leqslant \varepsilon_{n}\left\|y-P_{n} y\right\|_{2},
$$

where $\varepsilon_{n} \rightarrow 0$, as $n \rightarrow \infty$.

Now it is well known that $y_{n}^{\mathrm{I}}$ also exists for large enough $n$, and that $\left\|y-y_{n}^{\mathrm{I}}\right\|_{2}$ approaches zero with an order of convergence that is asymptotically the same as that of $\left\|y-P_{n} y\right\|_{2}$. Hence it follows that $\left\|y-y_{n}^{\mathrm{II}}\right\|_{2}$ converges more quickly to zero, by at least a factor of $\varepsilon_{n}$, than $\left\|y-y_{n}^{\mathrm{I}}\right\|_{2}$. This "improvement by iteration" has particular practical significance since the calculation of $y_{n}^{\text {II }}$ requires roughly the same amount of computation time as the calculation of $y_{n}^{\mathrm{I}}$ [28]. The obviously interesting mathematical problem, therefore, is: What is the order of the improvement in accuracy obtained by using $y_{n}^{\text {II }}$ as an approximation to $y$ rather than $y_{n}^{\mathrm{I}}$ ? We shall 
consider this problem for the particular case when $U_{n}$ is a certain space of spline functions, which we now define.

For any $n \in \mathbf{N}$, let $\Pi_{n}$ denote the mesh (partition) given by

$$
\Pi_{n}: 0=x_{0}<x_{1}<x_{2}<\cdots<x_{n}=1 \text {. }
$$

For $r \in \mathbf{N}$ and $\nu \in \mathbf{N}_{0}$, with $\nu<r$, we shall let $S_{r}^{\nu}\left(\Pi_{n}\right)$ denote the space of splines on $[0,1]$ which have order $r$, continuity $\nu$, and knots $\Pi_{n}$. Thus $u \in S_{r}^{\nu}\left(\Pi_{n}\right)$ if $u$ has $\nu-1$ continuous derivatives on $[0,1]$, and $u$ is a polynomial of degree not greater than $r-1$ on each $\left(x_{i-1}, x_{i}\right]$, for $i=1, \ldots, n$. When $\nu=0$, the splines are possibly discontinuous at the knot points, but, to ensure that they are well defined, we assume left continuity at each knot and right continuity at 0 .

Throughout the remainder of this paper $y_{n}^{\mathrm{I}}$ and $y_{n}^{\mathrm{II}}$ will denote the approximations to $y$ defined by (2.1) and (2.3), where

$$
U_{n}=S_{r}^{\nu}\left(\Pi_{n}\right), \quad n \in \mathbf{N}
$$

for some fixed $r \in \mathbf{N}$ and $\nu \in \mathbf{N}_{0}$, with $\nu<r$. It will be necessary in Section 5 to make a further restriction on the choice of $\nu$. We shall give our order of convergence estimates in terms of the maximum mesh spacing $h$, defined by

$$
h=\max _{i=1, \ldots, n}\left(x_{i}-x_{i-1}\right) \text {. }
$$

We note that for a uniform mesh we have $h=1 / n$.

The following quantitative estimates in the uniform norm have been derived by Chandler [8], [9].

THEOREM 2 (CHANDLER). If $k$ and $f$ are sufficiently smooth (for precise requirements see [8] or [9]), and if, as $n$ varies, the meshes $\Pi_{n}$ satisfy a certain quasiuniformity condition (see Section 4), then

$$
\left\|y-y_{n}^{\mathrm{I}}\right\|_{\infty}=O\left(h^{r}\right)
$$

and

$$
\left\|y-y_{n}^{\mathrm{II}}\right\|_{\infty}=O\left(h^{2 r}\right) .
$$

Remarks. (i) The estimates (2.6), (2.7) demonstrate the great improvement of $y_{n}^{\text {II }}$ over $y_{n}^{\mathrm{I}}$ when all our given information is sufficiently smooth. It is usually said that $y_{n}^{\text {II }}$ exhibits "global superconvergence" (since the best uniform approximation to a smooth $y$ from splines of order $r$ is generally only $O\left(h^{r}\right)$ ). If weak singularities are present in $k$ or $f$, however, the regularity requirements of Theorem 2 will not be satisfied (see, for example, [8, p. 106]), and estimates of $\left\|y-y_{n}^{\mathrm{I}}\right\|_{\infty}$ and $\left\|y-y_{n}^{\mathrm{II}}\right\|_{\infty}$ are not yet available. Such estimates will be obtained in Sections 4 and 5 of this paper.

(ii) An elegant overview and further development of the theory of superconvergence for equations with smooth and Green's function kernels are given in Chatelin and Lebbar [11], [12].

3. Regularity and Approximation. In this section we present two important theoretical results. The first (Theorem 3 below) describes how weak singularities in the kernel or inhomogeneous term of (1.1) affect the behavior of the solution, $y$. The second (Theorem 4) gives some spline approximation properties of typical weakly singular functions. 
These theoretical developments are facilitated by the introduction of certain Banach function spaces as follows. For $m \in \mathbf{N}$ and $1 \leqslant p \leqslant \infty$, we define $W_{p}^{m}[0,1]$ to be the usual Sobolev space of functions which have $m$ th derivative lying in $L_{p}[0,1]$. We assume that this space is normed in the usual way [19, Chapter 5]. For $\alpha>0$ and $1 \leqslant p \leqslant \infty$, we define the Nikol'skii space $N_{p}^{\alpha}[0,1]$ by

$$
N_{p}^{\alpha}[0,1]=\left\{\phi \in L_{p}[0,1]:|\phi|_{\alpha, p}:=\sup _{h \neq 0} \frac{\left\|\Delta_{h}^{2} \phi^{[\alpha]}\right\|_{L_{p}[0,1]_{2 h}}}{|h|^{\alpha_{0}}}<\infty\right\},
$$

where, for any $\varepsilon \in \mathbf{R}$,

$$
[0,1]_{\varepsilon}:=\{t \in[0,1]: t+\varepsilon \in[0,1]\},
$$

where $[\alpha] \in \mathbf{N}_{0}$ and $0<\alpha_{0} \leqslant 1$ are chosen to satisfy the equation

$$
\alpha=[\alpha]+\alpha_{0},
$$

and where $\Delta_{h}$ stands for the usual forward difference operator. $N_{p}^{\alpha}[0,1]$ is then a Banach space under the norm

$$
\|\phi\|_{\alpha, p}=\|\phi\|_{p}+|\phi|_{\alpha, p} .
$$

Remark. All the derivatives considered here are defined in the distributional sense [19, Section 5.3].

Fuller details of the motivation behind the introduction of the Nikol'skii spaces may be obtained by consulting [9] or [17]. However, their relevance to the analysis of weak singularities may be clarified by remarking that the function $t^{\alpha-1}$ is in $N_{1}^{\alpha}[0,1]$ but is not in $N_{1}^{\beta}[0,1]$, for any $\beta>\alpha$, and that $\ln t \in N_{1}^{1}[0,1]$. In addition, we point out the existence of the following continuous imbeddings [19, pp. 383-384, pp. 389-391]:

$$
N_{p}^{m+\varepsilon}[0,1] \subseteq W_{p}^{m}[0,1] \subseteq N_{p}^{m}[0,1] \subseteq N_{p}^{m-\varepsilon}[0,1],
$$

for $m \in \mathbf{N}, 0<\varepsilon<1$, and $1 \leqslant p \leqslant \infty$, and

$$
N_{p}^{\alpha}[0,1] \subseteq N_{q}^{\beta}[0,1],
$$

for $\alpha>0,1 \leqslant p \leqslant q \leqslant \infty$, and $\beta=\alpha-(1 / p-1 / q)>0$. The chain of imbeddings (3.1) demonstrates the fact that the Sobolev spaces are naturally immersed in the continuum of Nikol'skir spaces, while (3.2) shows that, given a function in a certain Nikol'skiǐ space, we may trade in some of its differentiability to obtain some stronger integrability properties.

We shall also use the space $C^{m}([0,1] \times[0,1])\left(m \in \mathbf{N}_{0}\right)$ of continuous functions on $[0,1] \times[0,1]$ whose mixed partial derivatives of any order $k$ with $|k| \leqslant m$ exist, are bounded, and are uniformly continuous on $(0,1) \times(0,1)[19$, p. 22].

We now introduce the assumptions:

Al. The kernel $k$ of (1.2) has the specific form

$$
k(t, s)=m(t, s) k(t-s), \quad t, s \in[0,1],
$$

with $k \in N_{1}^{\alpha}[-1,1]$ for some $1>\alpha>0$, and $m \in C^{2}([0,1] \times[0,1])$.

A2. The homogeneous equation

$$
y(t)=\int_{0}^{1} m(t, s) k(t-s) y(s) d s, \quad t \in[0,1],
$$

has no nontrivial solutions in $L_{1}[0,1]$. 
Then we have

THEOREM 3. Suppose A1 and A2 are satisfied.

(i) If $f \in C[0,1]$, then $y=(I-K)^{-1} f$ is well defined in $C[0,1]$.

(ii) If $f \in N_{1}^{\beta+1}[0,1]$, for some $\beta>0$, then $y \in N_{1}^{\min \{\alpha+1, \beta+1\}}[0,1]$.

Proof. It is well known that the operator $K$ given by $\mathrm{Al}$ is compact on $C[0,1]$. The proof of (i) follows by the Fredholm Alternative. To obtain (ii), we refer to Chandler [9], where it is shown that we have the chain of mappings

$$
W_{1}^{1}[0,1] \underset{K}{\rightarrow} N_{1}^{\alpha+1}[0,1] \underset{\text { Inclusion }}{\rightarrow} W_{1}^{1}[0,1]
$$

with $K$ bounded and the inclusion compact. Hence $K$ is compact as an operator on $W_{1}^{1}[0,1]$. Since, by (3.1), we have $f \in N_{1}^{\beta+1}[0,1] \subseteq W_{1}^{1}[0,1]$, it follows, by the Fredholm Alternative, that $y \in W_{1}^{1}[0,1]$. Consequently, by (3.3), we have $K y \in$ $N_{1}^{\alpha+1}[0,1]$ and hence, since $y=f+K y$, the result follows.

Some spline approximation properties of the Nikol'skii spaces are given in Theorem 4 below. A proof may be obtained by a trivial modification of the proof of Theorem 4.4 of [17].

THEOREM 4. Let $r \in \mathbf{N}, \nu \in \mathbf{N}_{0}$ be fixed with $\nu<r$.

(i) Let Al be satisfied. Then, for each $t \in[0,1]$, there exists a spline $u_{t} \in S_{r}^{\nu}\left(\Pi_{n}\right)$ such that

$$
\int_{0}^{1}\left|m(t, s) k(t-s)-u_{t}(s)\right| d s \leqslant C h^{\alpha},
$$

with $C$ independent of $t$ and $h$.

(ii) Let $\phi \in N_{\infty}^{\eta}[0,1] \cap C[0,1]$ for some $1>\eta>0$. Then there exists a spline $v \in S_{r}^{\nu}\left(\Pi_{n}\right)$ such that

$$
\|\phi-v\|_{\infty} \leqslant C h^{\eta}
$$

and $C$ is independent of $h$.

Discussion. The class of equations considered here is more general than that of [17, Chapter 4]. In the latter work we treated equations satisfying $\mathrm{Al}$ with $m \equiv 1$, and with $k \in N_{1}^{\alpha}[-1,1]$ for any $\alpha>0$. The inclusion of a smooth factor $m(t, s)$ into the kernel increases greatly the relevance of the theory to the type of weakly singular equations that arise in practice (see [17] and [25] for some examples). The fact that here we consider only $0<\alpha<1$ represents no restriction over [17] (since $N_{1}^{\alpha+\varepsilon}[0,1]$ $\subseteq N_{1}^{\alpha}[0,1]$ for $\varepsilon>0$ ), but does allow us to prove simpler orders of convergence for our numerical methods (cf. [17] where complicated logarithmic factors occur when $\alpha \in \mathbf{N})$.

4. Order of Convergence Estimates. In this section we derive global order of convergence estimates for the Galerkin and iterated Galerkin approximants to the solution of (1.1), when the kernel satisfies A1 and A2. The first step in proving the required estimates is given in Theorem 5 below, and consists of transforming the original convergence theory (Theorem 1 , and its sequel), from its $L_{2}[0,1]$ setting into a $C[0,1]$ setting. 
As in Theorem 2, we shall assume that, as $n$ varies, the partitions $\Pi_{n}$, used in the definitions of the splines, remain quasiuniform, i.e., there exists a constant $C$ with the property that

$$
\frac{\max _{i=1, \ldots, n}\left(x_{i}-x_{i-1}\right)}{\min _{j=1, \ldots, n}\left(x_{j}-x_{j-1}\right)} \leqslant C
$$

for each partition $\Pi_{n}$. We note that condition (4.1) implies that $h \rightarrow 0$ as $n \rightarrow \infty$, where $h$ is the maximum mesh spacing. It also follows from (4.1), [6], [14], that $P_{n}$ is bounded when considered as an operator on $L_{\infty}[0,1]$, and, in fact, there exists a constant $C$, independent of $n$, such that

$$
\left\|P_{n}\right\| \leqslant C, \quad n \in \mathbf{N} .
$$

Hence $P_{n}$ is then bounded as an operator from $C[0,1]$ to $L_{\infty}[0,1]$, with norm also satisfying (4.2).

Remarks. 1. It has recently been shown by Güsmann [18] that (4.2) will hold under substantially weaker (but more complicated) assumptions than (4.1). The treatment here omits this extra generality in the interests of simplicity.

2. If we restrict attention to splines in $S_{r}^{\nu}\left(\Pi_{n}\right)$ with $r=1$ and $\nu=0$ or $r \geqslant 2$ and $\nu \in\{0,1\}$, then (4.2) and the results of this section hold, irrespective of any conditions on $\Pi_{n}$. This fact is discussed in more detail in Section 5.

3. Chatelin and Lebbar [12] have shown how to obtain superconvergence results, without the requirement (4.1) for the numerical solution of equations with smooth (or Green's function) kernels using piecewise polynomial (i.e., $S_{r}^{0}\left(\Pi_{n}\right)$ ) approximation.

TheOREM 5. Let A1, A2 be satisfied, let $f \in C[0,1]$, and suppose that

$$
\left\|K-K P_{n}\right\|_{C[0,1]} \rightarrow 0, \text { as } n \rightarrow \infty \text {. }
$$

Then, for sufficiently large $n, y_{n}^{\mathrm{I}}, y_{n}^{\mathrm{II}}$ are well defined, $y_{n}^{\mathrm{I}} \in L_{\infty}[0,1], y_{n}^{\mathrm{II}} \in C[0,1]$.

$$
C_{1}\left\|y-P_{n} y\right\|_{\infty} \leqslant\left\|y-y_{n}^{\mathrm{I}}\right\|_{\infty} \leqslant C_{2}\left\|y-P_{n} y\right\|_{\infty},
$$

and

$$
\left\|y-y_{n}^{\mathrm{II}}\right\|_{\infty} \leqslant C\left\|K y-K P_{n} y\right\|_{\infty},
$$

with $C_{1}, C_{2}$, and $C$ independent of $n$.

Proof. We first consider $y_{n}^{\text {II }}$ and apply the collectively compact operator approximation theory of Anselone [1, Theorem 1.6].

Note first that it follows from [15] that $K$ is compact from $L_{\infty}[0,1]$ to $C[0,1]$, and so $K P_{n}$ is compact on $C[0,1]$. We also know, directly from (4.3), that $K P_{n} \rightarrow K$ pointwise on $C[0,1]$. It is straightforward to show [17], that the set

$$
\left\{K P_{n} \phi: n \in \mathbf{N}, \phi \in C[0,1],\|\phi\|_{\infty} \leqslant 1\right\}
$$

has compact closure in $C[0,1]$, and hence the set $\left\{K P_{n}: n \in \mathbf{N}\right\}$ is a collectively compact set of operators on $C[0,1]$. Then, since by Theorem 3(i), $(I-K)^{-1}$ exists on $C[0,1]$, it follows [1] that $\left(I-K P_{n}\right)^{-1}$ also exists on $C[0,1]$, for sufficiently large $n$, and is uniformly bounded in $n$. Using (2.4), it then follows that $y_{n}^{\text {II }}$ exists for large enough $n$, with

$$
y_{n}^{\mathrm{II}}=\left(I-K P_{n}\right)^{-1} f,
$$


and, by Theorem 3(i),

$$
y-y_{n}^{\mathrm{II}}=\left[(I-K)^{-1}-\left(I-K P_{n}\right)^{-1}\right] f=\left(I-K P_{n}\right)^{-1}\left(K-K P_{n}\right) y
$$

from which (4.5) follows.

Now return to $y_{n}^{I}$, the existence of which is guaranteed, for sufficiently large $n$, by (2.3). To obtain (4.4), we first use (2.3) and (4.6) to write

$$
\begin{aligned}
y-y_{n}^{\mathrm{I}} & =y-P_{n} y_{n}^{\mathrm{II}}=\left(y-P_{n} y\right)+P_{n}\left(y-y_{n}^{\mathrm{II}}\right) \\
& =\left(y-P_{n} y\right)+P_{n}\left(I-K P_{n}\right)^{-1}\left(K-K P_{n}\right) y,
\end{aligned}
$$

and, on using the known properties of $P_{n},\left(I-K P_{n}\right)^{-1}$ and $K$, we have

$$
\left\|y-y_{n}^{\mathrm{I}}\right\|_{\infty} \leqslant\left[1+\left\|P_{n}\right\|\left\|\left(I-K P_{n}\right)^{-1}\right\|\|K\|\right]\left\|y-P_{n} y\right\|_{\infty},
$$

and the right-hand inequality in (4.4) follows. Also, in view of (2.1), we may write

$$
\left(I-P_{n} K\right)\left(y-y_{n}^{\mathrm{I}}\right)=y-P_{n} K y-P_{n} f=y-P_{n} y,
$$

from which, using the known properties of $P_{n}$ and $K$, we obtain

$$
\left\|y-P_{n} y\right\|_{\infty} \leqslant[1+C\|K\|]\left\|y-y_{n}^{\mathrm{I}}\right\|_{\infty},
$$

with $C$ independent of $n$, and the left-hand inequality in (4.4) follows, completing the proof.

It is clear that, in order to satisfy (4.3) and to estimate the order of the right-hand side of (4.5), we must estimate $\left\|K \phi-K P_{n} \phi\right\|_{\infty}$, for any $\phi \in C[0,1]$. This is the purpose of the next theorem. In fact, it turns out that (4.3) is a redundant assumption, being automatically satisfied by Al. From now on, when proving convergence results, we shall let $C$ denote a generic constant.

THEOREM 6. Let Al be satisfied. Then, for $\phi \in C[0,1]$, we have

$$
\left\|\left(K-K P_{n}\right) \phi\right\|_{\infty} \leqslant C h^{\alpha}\left\|\phi-P_{n} \phi\right\|_{\infty} .
$$

Proof. For $t \in[0,1], n \in \mathbf{N}$, and $\phi \in C[0,1]$, we have, using Theorem 4(i) and the duality arguments of Chandler [7],

$$
\begin{aligned}
\left|\left(K-K P_{n}\right) \phi(t)\right| & =\left|\int_{0}^{1} m(t, s) k(t-s)\left(\phi(s)-P_{n} \phi(s)\right) d s\right| \\
& =\left|\int_{0}^{1}\left(m(t, s) k(t-s)-u_{t}(s)\right)\left(\phi(s)-P_{n} \phi(s)\right) d s\right| \\
& \leqslant \int_{0}^{1}\left|m(t, s) k(t-s)-u_{t}(s)\right| d s\left\|\phi-P_{n} \phi\right\|_{\infty},
\end{aligned}
$$

where we have used Hölder's inequality. The required estimate then follows from Theorem 4(i).

Corollary 7. Let A1, A2 be satisfied, and let $f \in C[0,1]$. Then, for sufficiently large $n, y_{n}^{\mathrm{I}}$ and $y_{n}^{\mathrm{II}}$ are well defined, (4.4) holds, and

$$
\left\|y-y_{n}^{\mathrm{II}}\right\|_{\infty} \leqslant C h^{\alpha}\left\|y-P_{n} y\right\|_{\infty} .
$$

Proof. It follows by Theorem 6 and (4.2) that, for $\phi \in C[0,1]$,

$$
\left\|\left(K-K P_{n}\right) \phi\right\|_{\infty} \leqslant C h^{\alpha}\|\phi\|_{\infty} .
$$


Hence (4.3) holds, and the estimates (4.4) and (4.5) follow. The required estimate for $y_{n}^{\text {II }}$ follows on application of Theorem 6 to (4.5).

The main theorem of this section (Theorem 8 below) estimates the rates of convergence of $y_{n}^{\mathrm{I}}$ and $y_{n}^{\mathrm{II}}$ to $y$, assuming the conditions of Corollary 7 and allowing $f$ to be continuous (but with possibly singular derivatives). The results are obtained by estimating $\left\|y-P_{n} y\right\|_{\infty}$ and using Corollary 7 .

We remark that the assumption

$$
f \in N_{1}^{\beta+1}[0,1], \text { for some } 1>\beta>0,
$$

leads via (3.1) to the conclusion that $f \in W_{1}^{1}[0,1]$, and hence $f$ is equivalent (i.e., equal almost everywhere) to an absolutely continuous function. In the following theorem the assumption (4.7) will be taken implicitly to mean that

$$
f \in W_{1}^{1}[0,1] \cap C[0,1] \text {. }
$$

TheOrem 8. Let A1, A2 and (4.7) be satisfied. Then the conclusions of Corollary 7 hold and

$$
\left\|y-y_{n}^{\mathrm{I}}\right\|_{\infty}=O\left(h^{\gamma}\right), \quad\left\|y-y_{n}^{\mathrm{II}}\right\|_{\infty}=O\left(h^{\gamma+\alpha}\right),
$$

where $\gamma=\min \{\alpha, \beta\}$.

Proof. Using (4.8) we deduce that the conclusions of Corollary 7 hold. By Theorem 3(ii) and (3.2), we have

$$
y \in N_{1}^{\min \{\alpha+1, \beta+1\}}[0,1] \subseteq N_{\infty}^{\min \{\alpha, \beta\}}[0,1],
$$

and by (4.8) and Theorem 3(i) it follows that $y$ is also in $C[0,1]$. Hence, using (4.2) we have, for any $\xi_{n} \in S_{r}^{\nu}\left(\Pi_{n}\right)$,

$$
\left\|y-P_{n} y\right\|_{\infty}=\left\|\left(I-P_{n}\right) y\right\|_{\infty}=\left\|\left(I-P_{n}\right)\left(y-\xi_{n}\right)\right\|_{\infty} \leqslant(1+C)\left\|y-\xi_{n}\right\|_{\infty},
$$

and thus, by Theorem 4(ii), $\left\|y-P_{n} y\right\|_{\infty} \leqslant C h^{\gamma}$, with $\gamma=\min \{\alpha, \beta\}$. The required estimates follow via Corollary 7.

5. A Graded Mesh. The results of Section 4 demonstrate that Galerkin methods for equations with singularities may sometimes possess rather poor rates of convergence. It may be remarked, however, that these poor rates arise partly as a result of our (rather naive) approach of using splines defined on arbitrary (quasiuniform) meshes, and that much better results may be obtained by using meshes which are specially graded to take account of the singularities in the solution.

In order to apply some known results concerning the use of graded meshes, we make the following more specific assumption on the integral operator $K$.

$\mathrm{Al}^{\prime}$. For some $0<\alpha \leqslant 1, \mu \in \mathbf{N}$, we have

$$
k(t, s)=m(t, s) k_{\alpha}(t-s),
$$

with $m \in C^{\mu+1}([0,1] \times[0,1])$ and

$$
\begin{aligned}
& k_{\alpha}(x)=|x|^{\alpha-1}, \quad 0<\alpha<1, \\
& k_{1}(x)=\ln |x| .
\end{aligned}
$$

Clearly any kernel which satisfies $A l^{\prime}$ for $0<\alpha<1$ will also satisfy $\mathrm{Al}$ for that value of $\alpha$. In addition, any kernel which satisfies A1' with $\alpha=1$ will also satisfy A1 for any $\alpha$ in the range $0<\alpha<1$. (This last fact follows from (3.1) since $\ln t \in$ $N_{1}^{1}[0,1]$; see [17].) 
The reason for introducing $\mathrm{Al}^{\prime}$ is that we may use a regularity result of Schneider [24] (Theorem 9 below), which is formulated specifically with applications to graded meshes in mind. Schneider's result utilizes the following concept, first introduced by Rice [21].

Definition. For $0<\beta<1, \mu \in \mathbf{N}$, we say that a function $\phi \in C[0,1]$ is of type $(\beta, \mu,\{0,1\})$ if

(i) $\left|\phi(t)-\phi\left(t^{\prime}\right)\right| \leqslant C_{1}\left|t-t^{\prime}\right|^{\beta}, t, t^{\prime} \in[0,1]$,

(ii) $\phi$ has $\mu$ continuous derivatives on $(0,1)$ which satisfy, for $i=1, \ldots, \mu$,

$$
\left|\phi^{(i)}(t)\right| \leqslant C_{2} \begin{cases}t^{\beta-i}, & 0<t \leqslant \frac{1}{2} \\ (1-t)^{\beta-i}, & \frac{1}{2} \leqslant t<1\end{cases}
$$

where $C_{1}$ and $C_{2}$ are independent of $t$ and $t^{\prime}$.

Then the following theorem follows from the arguments of [24].

TheOREM 9 (SCHNEIDER). Let $\mathrm{Al}^{\prime}$ and $\mathrm{A} 2$ be satisfied, and let $f$ be of type $(\beta, \mu,\{0,1\})$ for some $1>\beta>0, \mu \in \mathbf{N}$. Then $y$ is of type $(\gamma, \mu,\{0,1\})$, where $\gamma=\min \{\alpha, \beta\}$.

A similar result has been proved by Bechlars [5].

Now, consider the numerical solution of (1.1) under the conditions of Theorem 9. From now on our choice of approximating subspace $U_{n}$ is further restricted than that given by (2.5). In fact we choose

$$
U_{n}=S_{r}^{\nu}\left(\Pi_{n}\right) \text {, }
$$

with $r=1$ and $\nu=0$, or $r \geqslant 2$ and $\nu \in\{0,1\}$. So from now on our splines will either be piecewise continuous or (at the smoothest) elements of $C[0,1]$. The mesh $\Pi_{n}$ is no longer chosen to be arbitrary quasiuniform but rather is given (see Rice [21]) by

$$
\begin{cases}x_{i}=\frac{1}{2}(2 i / n)^{q}, & 0 \leqslant i \leqslant n / 2, \\ x_{i}=1-x_{n-i}, & n / 2 \leqslant i \leqslant n,\end{cases}
$$

where $q=r / \gamma$, with $\gamma=\min \{\alpha, \beta\}$. (Here we have corrected an error in [17, p. 92] where the wrong expression for the graded mesh (5.2) was given.) Note that the knots of this mesh are "bunched up" near the end points 0 and 1 (where $y$ behaves badly), and "spread out" in the interior of the interval $[0,1]$ (where $y$ is wellbehaved).

Then, with $U_{n}$ given by (5.1), (5.2), and provided the conditions of Theorem 9 are satisfied for $\mu \geqslant r$, it follows [21] that there exists $\xi_{n} \in U_{n}$ with the property that

$$
\left\|y-\xi_{n}\right\|_{\infty}=O\left(1 / n^{r}\right) \text {. }
$$

The mesh (5.2) does not satisfy the quasiuniformity requirement (4.1). However, in the case when $U_{n}$ is given by (5.1), the uniform boundedness condition (4.2) still holds regardless of how we choose our meshes. The case $\nu=1, r \geqslant 2$ (a result of Dupont) has been proved by de Boor in [6]. The case $\nu=0, r \geqslant 1$ can be proved as follows. Let $u \in L_{\infty}[0,1]$. Then, for $i=1, \ldots, n$, we have

$$
\left\|P_{n} u\right\|_{\infty}=\max _{i=1, \ldots, n}\left\|P^{(i)} u_{i}\right\|_{L_{\infty}\left[x_{i-1}, x_{i}\right]}
$$


where $u_{i}$ is the restriction of $u$ to $\left[x_{i-1}, x_{i}\right]$, and $P^{(i)}$ is the orthogonal projection onto the space of polynomials of degree less than $r$ defined on $\left[x_{i-1}, x_{i}\right]$. Then from [30, p. 236], we have for $i=1, \ldots, n$,

$$
\left\|P^{(i)} u_{i}\right\|_{L_{\infty}\left[x_{i-1}, x_{i}\right]} \leqslant C\left(x_{i}-x_{i-1}\right)^{-1 / 2}\left\|P^{(i)} u_{i}\right\|_{L_{2}\left[x_{i-1}, x_{i}\right]},
$$

with $C$ dependent only on $r$. Since $P^{(i)}$ is an orthogonal projection, we have

$$
\left\|P^{(i)} u_{i}\right\|_{L_{2}\left[x_{i-1}, x_{i}\right]} \leqslant\left\|u_{i}\right\|_{L_{2}\left[x_{i-1}, x_{i}\right]} \leqslant\left(x_{i}-x_{i-1}\right)^{1 / 2}\left\|u_{i}\right\|_{L_{\infty}\left[x_{i-1}, x_{i}\right]}
$$

and the result follows on combination of (5.4), (5.5) and (5.6).

We remark also that it has recently been shown by Güsmann [18] that the result (4.2) holds for splines of any order and continuity defined on the graded mesh (5.2).

Thus we have the final result:

THEOREM 10. Let the conditions of Theorem 9 be satisfied with $\mu \geqslant r$. Then, with $U_{n}$ given by (5.1) and (5.2), we have

$$
\left\|y-y_{n}^{\mathrm{I}}\right\|_{\infty}=O\left(1 / n^{r}\right), \quad\left\|y-y_{n}^{\mathrm{II}}\right\|_{\infty}=O\left(1 / n^{r+\alpha}\right) .
$$

Proof. The assumptions imply that the estimates of Corollary 7 hold. For the meshes (5.2) it can be shown that there exists $C$ independent of $n$ such that

$$
\frac{1}{n}<h \leqslant C \frac{1}{n} \text {. }
$$

Also, in view of (5.3) and the fact that (4.2) holds, we have, for $\xi_{n} \in U_{n}$,

$$
\left\|y-P_{n} y\right\|_{\infty}=\left\|\left(I-P_{n}\right)\left(y-\xi_{n}\right)\right\|_{\infty} \leqslant C\left\|y-\xi_{n}\right\|_{\infty} \leqslant C \frac{1}{n^{r}} .
$$

Combining this and (5.7) with the estimates given in Corollary 7 completes the proof.

The estimates (1.6) and (1.7) are a particular case of this theorem.

6. Numerical Examples. In each of the following examples splines in $S_{1}^{0}\left(\Pi_{n}\right)$ (i.e., piecewise constant functions) were used as approximating subspaces. In Examples 1 and 2 a uniform mesh was used while in Example 3 a graded mesh was used. In order to get an accurate assessment of the experimental error, in each example we choose $f$ so that $y$ has a particularly simple closed form. To obtain theoretical convergence rates for Examples 1, 2, and 3 we use the known regularity and approximation properties of $y$ along with Corollary 7. For these examples we cannot use Theorems 8 and 10 to estimate theoretical rates of convergence. This is because these theorems employ the natural singularities that arise in $y$ when $f$ is given arbitrarily, and hence are not applicable when $f$ is specially chosen. However, in Examples 2 and 3 we do solve an equation with a nonsmooth solution, and so these examples, although specially chosen, are realistic indicators of how the experimental results would compare with the theory in more general cases. In Tables 1, 2, and 3 the estimated order of convergence, EOC, of the quantity $e_{n}$, say, was calculated using the formula

$$
\mathrm{EOC}=\frac{\ln \left(e_{n} / e_{2 n}\right)}{\ln 2}
$$


Example 1.

$$
y(t)=f(t)+\int_{0}^{1}|t-s|^{-1 / 2} y(s) d s, \quad t \in[0,1],
$$

where $f$ was chosen so that $y(t)=t$. Note that Al is satisfied with $\alpha=\frac{1}{2}$. Since the solution is contrived to be smooth, and since the mesh is uniform, we have

$$
\left\|y-P_{n} y\right\|_{\infty}=O(1 / n)
$$

and so Corollary 7 gives

$$
\left\|y-y_{n}^{\mathrm{I}}\right\|_{\infty}=O(1 / n)
$$

and

$$
\left\|y-y_{n}^{\mathrm{II}}\right\|_{\infty}=O\left(1 / n^{3 / 2}\right) .
$$

The results are shown in Table 1 .

TABLE 1

\begin{tabular}{|c||c|c|c|c|}
\hline $\mathrm{n}$ & $\| \mathrm{y}-\mathrm{y}_{\mathrm{n}}^{\mathrm{I}_{\infty}}$ & $\begin{array}{c}\text { EOC } \\
\text { (Theory } \\
\text { predicts 1.0) }\end{array}$ & $\left\|\mathrm{y}-\mathrm{y}_{\mathrm{n}}^{\mathrm{II}}\right\|_{\infty}$ & $\begin{array}{c}\text { EOC } \\
\text { (Theory } \\
\text { preducts 1.5) }\end{array}$ \\
\hline 2 & 0.47 & 1.31 & 0.32 & 1.54 \\
8 & 0.19 & 1.27 & 0.11 & 1.78 \\
16 & $0.79(-1)$ & 1.13 & $0.32(-1)$ & 1.71 \\
32 & $0.36(-1)$ & 1.08 & $0.98(-2)$ & 1.71 \\
\hline
\end{tabular}

Example 2.

$$
y(t)=f(t)+\lambda \int_{0}^{1}|t-s|^{-1 / 2} y(s) d s, \quad t \in[0,1]
$$

where $\lambda, f$ were chosen (see [25], [29]) so that $y(t)=2 \sqrt{2}[t(1-t)]^{3 / 4}$. This problem has been considered by several other authors [4], [25], [29], [33] and was also solved in [17] using its equivalent formulation as an equation on the interval $[-1,1]$. Al is satisfied with $\alpha=\frac{1}{2}$. The solution is not smooth, and in fact $y \in N_{1}^{7 / 4}[0,1] \subseteq$ $N_{\infty}^{3 / 4}[0,1] \cap C[0,1]$, and so Theorem 4(ii) implies that

$$
\left\|y-P_{n} y\right\|_{\infty}=O\left(1 / n^{3 / 4}\right) \text {. }
$$


Thus Corollary 7 predicts

$$
\left\|y-y_{n}^{\mathrm{I}}\right\|_{\infty}=O\left(1 / n^{3 / 4}\right) \text { and }\left\|y-y_{n}^{\mathrm{II}}\right\|_{\infty}=O\left(1 / n^{5 / 4}\right) .
$$

The results are shown in Table 2 .

TABLE 2

\begin{tabular}{|c||c|c||c|c|}
\hline $\mathrm{n}$ & $\left\|\mathrm{y}-\mathrm{y}_{\mathrm{n}}^{\mathrm{I}}\right\|_{\infty}$ & $\begin{array}{c}\text { EOC } \\
\text { (Theory } \\
\text { predicts 0.75) }\end{array}$ & $\left\|\mathrm{y}-\mathrm{y}_{\mathrm{n}}^{\mathrm{II}}\right\|_{\infty}$ & $\begin{array}{c}\text { EOC } \\
\text { (Theory } \\
\text { predicts 1.25) }\end{array}$ \\
\hline 2 & 0.75 & 0.35 & 0.40 & 0.57 \\
8 & 0.59 & 0.71 & 0.27 & 1.17 \\
16 & 0.36 & 0.78 & 0.12 & 1.29 \\
32 & 0.21 & 0.81 & $0.49(-1)$ & 1.29 \\
\hline
\end{tabular}

Example 3. We consider the same equation as in Example 2, but this time we use a graded mesh as described in Section 5. Since $y$ is of type $\left(\frac{3}{4}, \mu,\{0,1\}\right)$ for any $\mu \in \mathbf{N}$, and we are using splines of order 1 , we set the grading exponent $q=\frac{4}{3}$. Thus (5.8) holds with $r=1$, and, combining this with the estimates of Corollary 7, we obtain

$$
\left\|y-y_{n}^{\mathrm{I}}\right\|_{\infty}=O(1 / n) \text { and }\left\|y-y_{n}^{\mathrm{II}}\right\|_{\infty}=O\left(1 / n^{3 / 2}\right) .
$$

The results are shown in Table 3.

TABLE 3

\begin{tabular}{|c||c|c||c|c|}
\hline $\mathrm{n}$ & $\left\|\mathrm{y}-\mathrm{y}_{\mathrm{n}}\right\|_{\infty}$ & $\begin{array}{c}\text { EOC } \\
\text { (Theory } \\
\text { predicts 1.0) }\end{array}$ & $\| \mathrm{y}-\mathrm{y}_{\mathrm{n}} \mathrm{II}_{\infty}$ & $\begin{array}{c}\text { EOC } \\
\text { (Theory } \\
\text { predicts 1.5) }\end{array}$ \\
\hline 2 & 0.75 & 0.50 & 0.40 & 0.80 \\
4 & 0.53 & 0.92 & 0.23 & 1.31 \\
8 & 0.28 & 1.00 & $0.93(-1)$ & 1.54 \\
16 & 0.14 & 1.11 & $0.32(-1)$ & 1.68 \\
32 & $0.65(-1)$ & & $0.10(-1)$ & \\
\hline
\end{tabular}


7. Acknowledgements. The author wishes to thank Dr. Claus Schneider (Johannes Gutenberg-Universität, Mainz) and Professor Françoise Chatelin and M. Rachid Lebbar (Université de Grenoble) for their hospitality and interest in this work. He is also indebted to Professor Carl de Boor for a stimulating discussion on splines and for directing him to reference [18].

Department of Mathematics

University of Melbourne

Parkville, Victoria 3052, Australia

1. P. M. Anselone, Collectively Compact Operator Approximation Theory, Prentice-Hall, Englewood Cliffs, N. J., 1971.

2. P. M. ANSElone, "Singularity subtraction in the numerical solution of integral equations," $J$ Austral. Math. Soc. Ser. B., v. 22, 1981, pp. 408-418.

3. P. M. ANSElone \& W. Krabs, “Approximate solution of weakly singular integral equations," $J$. Integral Equations, v. 1, 1979, pp. 61-75.

4. C. T. H. BAKER, The Numerical Treatment of Integral Equations, Clarendon Press, Oxford, 1977.

5. J. BECHLARS, Glattheit und numerische Berechnung der Lösung linearer Integralgleichungen 2. Art mit schwachsingulären Kernen, Report HMI-B283, Hahn-Meitner-Institut für Kernforschung, Berlin GmbH, 1978.

6. C. DE BooR, "A bound on the $L_{\infty}$-norm of $L_{2}$-approximation by splines in terms of a global mesh ratio," Math. Comp., v. 30, 1976, pp. 765-771.

7. G. A. ChANDLER, Global Superconvergence of Iterated Galerkin Solutions for Second Kind Integral Equations, Technical Report, Australian National University, Canberra, 1978.

8. G. A. Chandler, "Superconvergence for second kind integral equations," Application and Numerical Solution of Integral Equations (R. S. Anderssen, F. R. de Hoog and M. A. Lukas, Eds.), Sijthoff and Noordhoff, Alphen aan den Rijn, 1980.

9. G. A. Chander, Superconvergence of Numerical Solutions to Second Kind Integral Equations, Ph. D. thesis, Australian National University, Canberra, 1979.

10. G. A. Chandler, Product Integration Methods for Weakly Singular Second Kind Integral Equations, Technical Report, Australian National University, Canberra, 1979.

11. F. Chatelin \& R. LebBaR, "The iterated projection solution for the Fredholm integral equation of second kind,” J. Austral. Math. Soc. Ser. B., v. 22, 1981, pp. 439-451.

12. F. Chatelin \& R. LebBaR, "Superconvergence results for the iterated projection method applied to a second kind Fredholm integral equation and eigenvalue problem." (Preprint.)

13. L. M. Delves, L. F. Abd-Elal \& J. A. Hendry, "A fast Galerkin algorithm for singular integral equations,” J. Inst. Math. Appl., v. 23, 1979, pp. 139-166.

14. J. Douglas, JR., T. Dupont \& L. Wahlbin, “Optimal $L_{\infty}$ error estimates for Galerkin approximations to solutions of two-point boundary value problems," Math. Comp., v. 29, 1975, pp. 475-483.

15. I. G. Graham \& I. H. Sloan, “On the compactness of certain integral operators," J. Math. Anal. Appl., v. 68, 1979, pp. 580-594.

16. I. G. Graham, "Singularity expansions for the solutions of second kind Fredholm integral equations with weakly singular convolution kernels," J. Integral Equations, v. 4, 1982, pp. 1-30.

17. I. G. Graham, The Numerical Solution of Fredholm Integral Equations of the Second Kind, Ph. D. thesis, University of New South Wales, Sydney, 1980.

18. B. GUSmanN, " $L_{\infty}$-bounds of $L_{2}$-projections on splines," Quantitative Approximation (R. A. De Vore and K. Scherer, Eds.), Academic Press, New York, 1980.

19. A. Kufner, O. John \& S. Fučik, Function Spaces, Noordhoff International, Leyden, 1977.

20. LIN QUN \& LIU JIAQUAN, “Extrapolation method for Fredholm integral equations with non-smooth kernels," Numer. Math., v. 35, 1980, pp. 459-464.

21. J. R. RICE, "On the degree of convergence of nonlinear spline approximation," Approximations with Special Emphasis on Spline Functions (I. J. Schoenberg, Ed.), Academic Press, New York, 1969.

22. G. R. Richter, “On weakly singular Fredholm integral equations with displacement kernels," $J$. Math. Anal. Appl., v. 55, 1976, pp. 32-42.

23. D. W. SCHLITT, "Numerical solution of a singular integral equation encountered in polymer physics," J. Math. Phys., v. 9, 1968, pp. 436-439. 
24. C. SCHNEIDER, "Regularity of the solution to a class of weakly singular Fredholm integral equations of the second kind," Integral Equations Operator Theory, v. 2, 1979, pp. 62-68.

25. C. SCHNEIDER, "Product integration for weakly singular integral equations," Math. Comp., v. 36, 1981, pp. 207-213.

26. I. H. SloAN, “Error analysis for a class of degenerate-kernel methods," Numer. Math., v. 25, 1976, pp. 231-238.

27. I. H. SLOAN, "Improvement by iteration for compact operator equations," Math. Comp., v. 30, 1976, pp. 758-764.

28. I. H. Sloan, B. J. Burn \& N. DatyneR, “A new approach to the numerical solution of integral equations," J. Comput. Phys., v. 18, 1975, pp. 92-105.

29. A. SPENCE, "Product integration for singular integrals and singular integral equations," Numerische Integration (G. Hämmerlin, Ed.), Internat. Ser. Numer. Math., vol. 45, Birkhauser Verlag, Basel, 1979.

30. A. F. Timan, Theory of Approximation of Functions of a Real Variable, Pergamon Press, New York, 1963.

31. G. VainikKo \& A. Pedas, “The properties of solutions of weakly singular integral equations," $J$. Austral. Math. Soc. Ser. B., v. 22, 1981, pp. 419-430.

32. G. Vainikxo \& P. UBA, "A piecewise polynomial approximation to the solution of an integral equation with weakly singular kernel," J. Austral. Math. Soc. Ser. B, v. 22, 1981, pp. 431-438.

33. W. VolK, Die numerische Behandlung Fredholm'scher Integralgleichungen zweiter Art mittels Splinefunktionen, Report HMI-B286, Hahn-Meitner-Institut für Kernforschung, Berlin GmbH, 1979. 